\title{
Behavioral Implications of Piezoelectric Stack Actuators for Control of Micromanipulation
}

NASA-CR-205000

\author{
Michael Goldfarb \\ Nikola Celanovic \\ Department of Mechanical Engineering \\ Vanderbilt University \\ Nashville, TN 37235
}

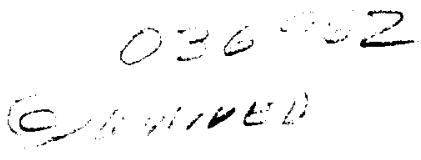

Abstract

A lumped-parameter model of a piezoelectric stack actuator has been developed to describe actuator behavior for purposes of control system analysis and design, and in particular for microrobotic applications requiring accurate position and/or force control. In addition to describing the input-output dynamic behavior, the proposed model explains aspects of non-intuitive behavioral phenomena evinced by piezoelectric actuators, such as the input-output rate-independent hysteresis and the change in mechanical stiffness that results from altering electrical load. The authors incorporate $a$ generalized Maxwell resistive capacitor as a lumpedparameter causal representation of rate-independent hysteresis. Model formulation is validated by comparing results of numerical simulations to experimental data.

\section{Introduction}

Piezoelectric ceramics transduce energy between the electrical and mechanical domains. Application of an electric field across the ceramic creates a mechanical strain, and in a similar manner, application of a mechanical stress on the ceramic induces an electrical charge. Since these devices are monolithic and have no sliding or rolling parts, they exhibit no significant mechanical stiction. A typical lead-zirconate-titanate (PZT) piezoelectric actuator, for example, can perform step movements with a resolution on the order of a nanometer and a bandwidth on the order of a kilohertz. Consequently, piezoelectric ceramics are well suited for use as precision microactuators for micropositioning devices or micromanipulators. Such applications require control design which can provide both accurate position tracking performance and suitable stability robustness. The purpose of the model presented herein is to map the relationship between voltage and charge at the electrical port of the PZT to force and displacement at the mechanical port in a lumped parameter form that can be represented by a finite number of ordinary differential equations. This type of formulation provides both general insight into PZT behavior as well as a specific causal mathematical representation for purposes of model-based control system analysis and design.

\section{The Standard on Piezoelectricity}

The most widely recognized description of piezoelectric ceramic behavior was published by a standards committee of the Institute of Electrical and Electronics Engineers [4]. The linearized constitutive relations formulated by this commitee essentially state that material strain and electrical displacement (charge per unit area) exhibited by a piezoelectric ceramic are both linearly affected by the mechanical stress and electrical field to which the ceramic is subjected. Several researchers have utilized the IEEE constitutive relations to derive a piezoelectric actuator model. Basing model derivation on the IEEE constitutive relations, however, requires several simplifying assumptions and fails to explicitly describe the extreme nonlinearities that are present in all piezoelectric ceramics, rendering the resulting description too approximate for use in developing suitable position or force control. Others have modelled the behavioral nonlinearities of piezoelectric ceramic with algorithmic methods that provide little physical insight into actuator behavior and have limited use with respect to model-based control system design.

\section{Model Formulation}

The fundamental component of a piezoelectric stack actuator is a wafer of piezoelectric material sandwiched between two electrodes. Prior to fabrication, the wafer is polarized uniaxially along its thickness, and thus exhibits significant piezoelectric effect in this direction only. A typical piezoelectric stack actuator is formed by assembling several of the wafer elements in series mechanically and connecting the electrodes so that 
the wafers are in parallel clectrically, as illustrated in Figure 1. Since piezoccramic is a known dielectric, one would expect such a configuration to exhibit capacitive behavior. The electrical behavior of a PZT stack actuator, however, is significandy more complex. For purposes of controller design, one of the most inconvenient aspects of the actuator behavior is the rateindependent hysteresis exhibited between voltage and displacement as well as between force and displacement, as shown in Figure 2. If not specifically addressed, this type of behavior can cause closed-loop limit cycling and possibly instability.

Experimental observation indicates that the rateindependent hysteresis exbibited in Figure 2 is not present between the endpoint displacement of the PZT stack actuator and the net electrical charge delivered to the actuator. Additionally, dynamic observation indicates that endpoint displacement as a function of electrical charge is well-approximated by second-order linear dynamics, as shown in the measured data of Figure 3.

The quasi-static force-displacement relationship of a PZT stack actuator is shown in Figure 4. As illustrated in the figure, rate-independent hysteresis is observed only when the electrode leads are shorted. When the leads are open and current cannot flow through the ceramic, the actuator exhibits no static hysteresis. This evidence, along with the absence of static bysteresis between displacement and charge illustrated by Figure 3(a), suggests that the rate-independent hysteresis lies solely in the electrical domain between the applied actuator voltage and resulting charge. The hysteresis can therefore be characterized by an electrical resistor with constitutive relations that, unlike a typical resistor, relate voltage to charge rather than current. This type of rateindependent dissipation is commonly experienced mechanically as Coulomb friction. This analogy is the basis for describing the static hysteresis exhibited by the piezoelectric actuator. Figure 5 illustrates a single elastoslide element which consists of a massless linear spring and a massless block that is subjected to Coulomb friction. For a displacement input of sufficient amplitude, the relationship between the applied force and the endpoint displacement will exhibit rudimentary hysteretic behavior. If several of these clasto-slide elements are put in parallel, each subjected to an incrementally larger normal force, the simple relationship of rigure 5 becomes a piccewise linear approximation of the continuous hysteresis exhibited by the pieroclectric actuator, as illustrated in liggure 6 . This construction wats initially formulated by the

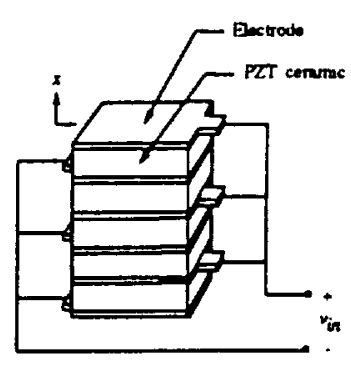

Figure 1. Ilustration of a piezoelectric stack actuator.
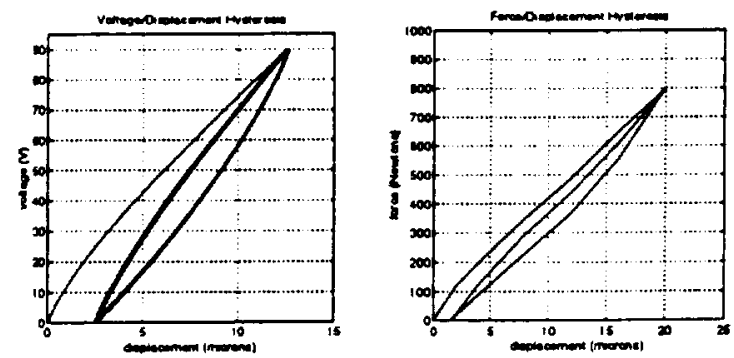

Figure 2. Measured quasi-static relationships between applied voltage and endpoint displacement and between applied force and endpoint displacement.
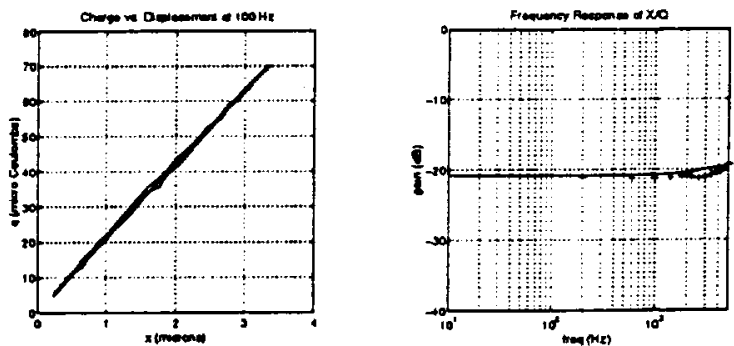

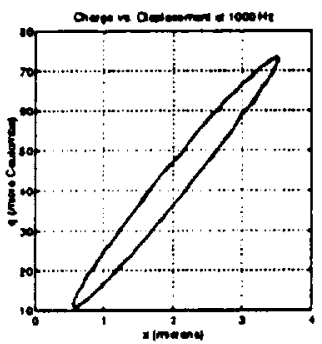

(a)

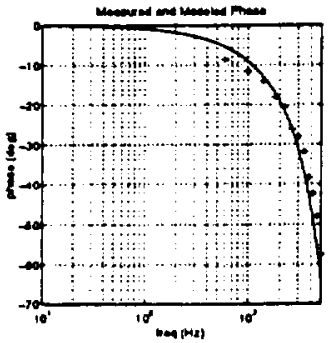

(b)
Figure 3. Column (a): Charge input versus endpoint displacement output for PZT stack actuator for 100 and $1000 \mathrm{~Hz}$ sinusoidal inputs.

Column (b): Frequency response of endpoint displacement output to actuator charge input. The marks represent measured data points and the continuous lines represent a lineur second-order system. 


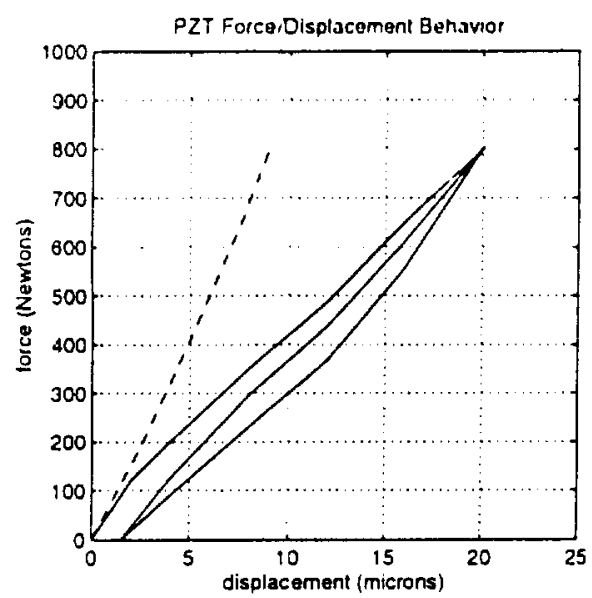

Figure 4. Quasi-static force displacement relationsbip for a PZT stack actuator with open leads (dashed line) and with closed leads (solid line).

mathematician and physicist James $C$. Maxwell in the mid-1800's, and in the limit as the number of elasto-slip elements becomes infinite, the model is referred to as Generalized Maxwell Slip [2]. Though originally a mechanical formulation, the energy-based constitutive relations of the Maxwell slip model are not domain specific, and can therefore represent any rateindependent hysteretic relationship between a generalized effort and generalized displacement in a lumped parameter causal form. Consequently, in addition to force and displacement, the generalized Maxwell model can represent rate-independent hysteresis between voltage and charge, temperature and entropy, and magnetomotive force and magnetic flux. Note also that the number of elasto-slip elements has no bearing on the order of the model, since the blocks are all massless and the springs are in parallel.

The PZT stack actuator model resulting from the aforementioned observations is shown in schematic form in Figure 7. The generalized Maxwell resistive capacitance, which is represented by the MRC element, resides in the electrical domain and therefore relates the element's electrical voltage to charge. The PZT model has two ports of interaction, a voltage-current port on the electrical side and a force-velocity port on the mechanical side. With respect to the mechanical side of the transformer, since the actuator model is concerned only with endpoint displacement in a band within the first mechanical mode of vibration, the piezoclectric stack is assumed to have a lumped masss and a linear matcrial stiffiness and damping.

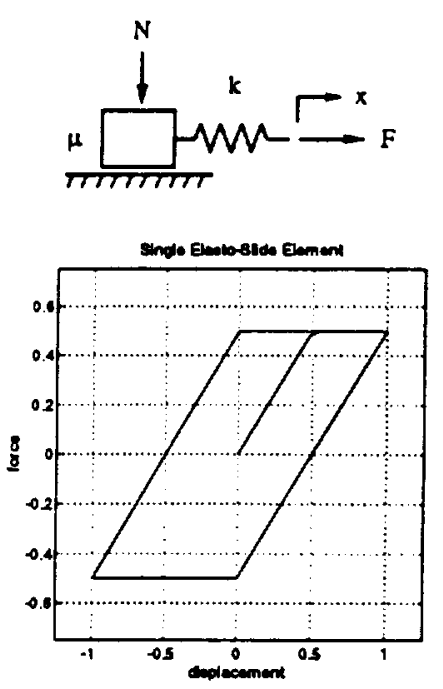

Figure 5. Force-displacement behavior of a single elastoslide element consisting of a massless linear spring and a massless block subjected to Coulomb friction.
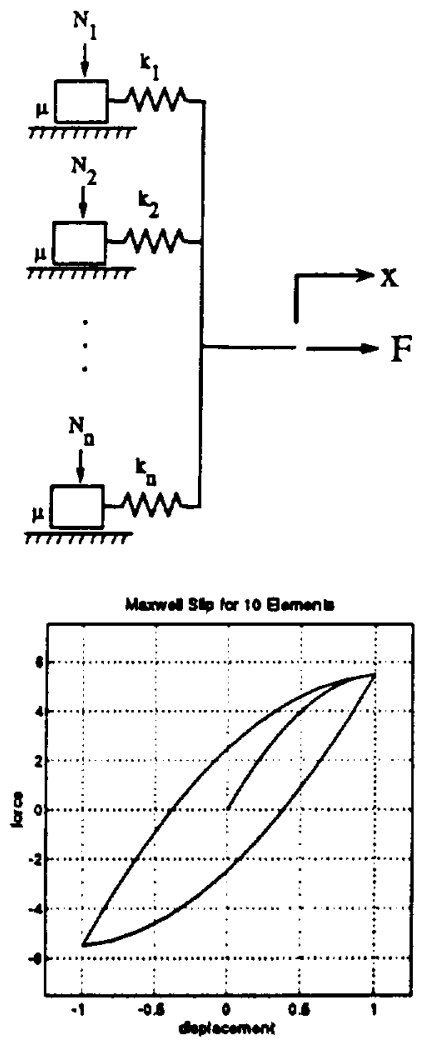

Figurc 6. Fore-displacement behavior of several clastoslide elements in parallel, each subjected to increasing normal forces. 
The behavior of the actuator is therefore described by:

$$
\begin{aligned}
& v_{i n}=v_{r}+v_{t} \\
& q=n x+C v_{t} \\
& m \ddot{x}+b \dot{x}+k x=F_{t}+F_{e x t} \\
& F_{t}=n v_{t}
\end{aligned}
$$

where $q$ is the total charge in the ceramic, $v_{i n}$ is the actuator input voltage, $v_{r c}$ is the voltage across the Maxwell capacitor (which is a function of $q$ ), $v_{t}$ is the back-emf from the mechanical side, $C$ is the linear capacitance in parallel with the transformer, $n$ is the electromechanical transformer ratio, $m, b$, and $k$ are the mass, damping, and stiffness of the ceramic, $x$ is the stack endpoint displacement, $F_{t}$ is the transduced force from the electrical domain, and $F_{e x x}$ is the force imposed from the external mechanical load.

\section{Simulation and Experiment}

The model was parameterized for a commercially available piezoelectric stack actuator (NEC model \#AE0505D16). This actuator operates at input voltages between zero and 150 volts (in the direction of wafer polarization), which corresponds to an endpoint displacement range of approximately 25 microns. Upon measuring the mass $m$ of the PZT, the mechanical stiffness $k$ and damping $b$ can be determined by observation of the charge-displacement dynamics that are shown in Figure 3. Measurement of the open-lead stiffness and knowledge of the DC gain between charge and displacement are sufficient to determine the linear electrical capacitance $C$ and the transformer ratio $n$. The model parameters utilized for the simulations that follow are given in Table 1. After determining the parameters of Table 1, the Maxwell capacitor parameters can be determined by propagating experimental results through the model. The parameters defining the Maxwell capacitor for the simulations presented herein are given in Table 2. As indicated by the table, these simulations were run with ten elasto-slip elements in the generalized Maxivell resistive capacitor. A real-time application may be better served by fewer elements; since the number of elements does not affect the order of the model, however, the increased computational overhead from added elements is minimal.

Figure 8 shows the measured and simulated endpoint displacement response of the modeled actuator to a 90 voll $100 \mathrm{~Hz}$ triangle-wave voltage input in the absence of any external mechanical load. $\Lambda s$ indicated by the plots, the model faithfully represents the voltage input to endpoint displaceinent output behavior of the

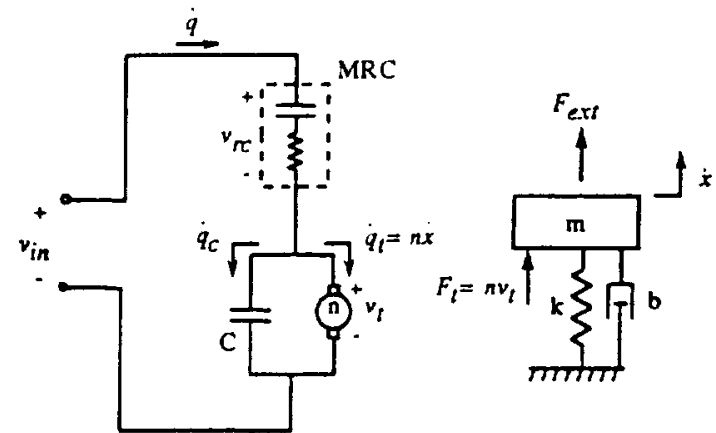

Figure 7. Schematic representation of the PZT stack

\begin{tabular}{|c|c|c|}
\hline $\begin{array}{l}\text { Model } \\
\text { Parameter }\end{array}$ & Symbol & Numerical Value \\
\hline mass & $m$ & $0.00375 \mathrm{~kg}$ \\
\hline stiffness & $k$ & $6 \times 10^{6} \quad \mathrm{~N} / \mathrm{m}$ \\
\hline darmping & $b$ & $150 \quad$ N-s/m \\
\hline $\begin{array}{l}\text { linear } \\
\text { capacitance }\end{array}$ & $C$ & $1.2 \times 10^{-6} \mathrm{~F}$ \\
\hline $\begin{array}{l}\text { transformer } \\
\text { ratio }\end{array}$ & $n$ & $\mathrm{C} / \mathbf{m}$ \\
\hline
\end{tabular}
actuator model. The capacitor and resistor contained within the MRC element are both nonlinear elements.

Table 1. Model parameters utilized in simulation of the NEC model \#AE0505D16 piezoelectric actuator.

\begin{tabular}{|c|c|c|}
\hline Element & Stimness $\left(\mu \mathrm{F}^{-1}\right)$ & Break Force $(V)$ \\
\hline$n$ & $k_{n}\left(1 / C_{n}\right)$ & $f_{n}=\mu N_{n}\left(V_{n}\right)$ \\
\hline 1 & 2.0 & 0.2 \\
\hline 2 & 0.6 & 0.3 \\
\hline 3 & 0.3 & 0.3 \\
\hline 4 & 0.26 & 2.6 \\
\hline 5 & 0.06 & 0.9 \\
\hline 6 & 0.1 & 2.0 \\
\hline 7 & 0.05 & 1.5 \\
\hline 8 & 0.03 & 1.2 \\
\hline 9 & 0.1 & 7.0 \\
\hline 10 & 0.5 & 80.0 \\
\hline
\end{tabular}

Table 2. Generalized Maxwell capacitor parameters utilized in the model. The stiffnesses and breakaway forces correspond with the mechanical schematic of Figure 6.

PZT actuator. Note in the plots that the displacement output evinces distortion on both the rising and falling slopes and maintains an amplitude-dependent offset. Further observation indicates that this is not typical dynamic distortion. There is no discernible phase lag between the input and output, as indicated by the relative positions of the waveform peaks, and there are no significant filtering effects, evideneed by the fact that the peaks are neither rounded nor otherwise distorted. These 
obscrvations indicate the existence of a rate-independent hysteresis. This hysteretic behavior is clearly displayed in Figure 9, which shows both the measured and simulated data of Figure 8 plotted as volage input versus displacement output. Any dynamic system will exhibit an ellipsoid-shaped input-output hysteresis, provided the input is of sufficient frequency to create discemible phase lag in the output. This type of dynamic hysteresis is characterized by a smooth curve relating the output to input, such as that shown in Figure 3. The rateindependent hysteresis of Figure 8, however, is distinguished from a dynamic hysteresis by the distinct discontinuities exhibited at both extremes. Model accuracy is additionally demonstrated by the data of Figure 10 , which shows the measured and simulated response of the actuator to a linearly decaying $100 \mathrm{Hertz}$ sinusoidal voltage input.
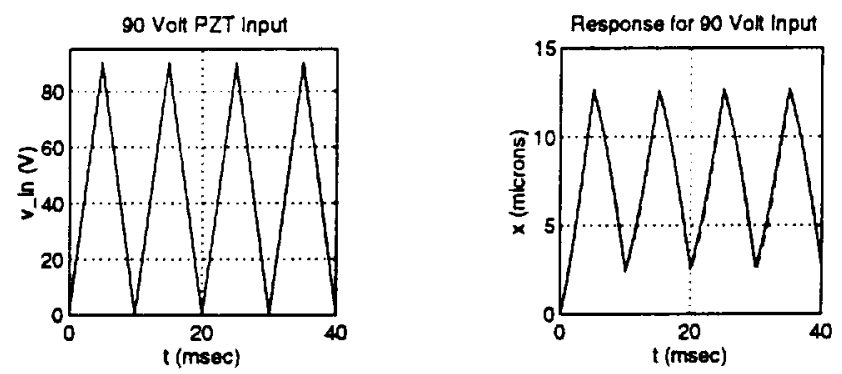

Figure 8. Measured (solid line) and simulated (dashed line) endpoint displacement response of the PZT to a 90 volt $100 \mathrm{~Hz}$ triangle-wave input. The difference between the measured and simulated data is difficult to discem.

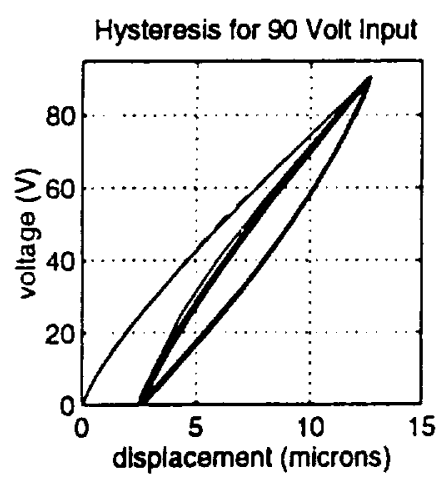

Figure 9. Measured (solid line) and simulated (dashed line) voltage versus displacement for a 90 volt $100 \mathrm{IIz}$ triangle-wave voltage input. The difference between the measured and simulated data is difficult to discern.
Figure 11 shows the simulated displacement response to a $10 \mathrm{II} z$ sinusoidal external force for cases with the electrical leads open and shorted. The simulated behavior accuratcly reflects the quasistatic measured behavior illustrated by Figure 4. In addition to providing a structure for numerical simulation, the lumpedparameter model also provides insight into how the electrical properties of the PZT reflect into the mechanical domain. As shown by the measured and simulated data of Figures 4 and 11 respectively, the PZT actuator exhibits significantly greater stiffness when the leads are open than when the leads are shorted. This change in stiffness can be demonstrated by observing that both the linear and the Maxwell capacitors in the electrical domain reflect as stiffnesses in the mechanical domain. This is similar to the electrical resistance of a DC motor appearing as mechanical damping when the motor leads are shorted. Linearizing the Maxwell capacitor and deriving expressions for mechanical stiffness in both the open and shorted-lead configurations yields

$$
\begin{gathered}
k_{o}=k+\frac{n^{2}}{C} \\
k_{s}=k+\frac{n^{2}}{C_{m}+C}
\end{gathered}
$$

where $k_{0}$ is the open lead stiffness, $k_{s}$ is the shorted lead stiffness, $k$ is the mechanical domain stiffness, $C$ is the linear electrical capacitance, $n$ is the transformer ratio, and $C_{m}$ is the linearized Maxwell capacitor. The inclusion of $C_{m}$ in the second term of the latter expression clearly indicates the decreased shorted lead stiffness.

\section{Implications for Actuator Control}

Comstock [1] and Newcomb and Flinn [3] observed that utilizing charge input to command endpoint displacement of a PZT stack actuator resulted in better closed-loop performance than when using voltage input. The model presented herein elucidates the reason for performance improvement. Controlling actuator charge prevents the nonlinear Maxwell capacitor from independently storing energy, and therefore effectively removes it from the input-output behavior of the plant. Controlling charge thus renders the PZT stack a linear second-order system. With respect to force control, the model indicates that endpoint force can be commanded directly by constraining the stack to have zero displacement and controlling the input charge. 
Though charge control of a PZT actuator circumvents the nonlinear behavior of piezoelectric ceramic and enables the use of linear control techniques. the simplicity of linear control is bought at the expense of the increased electronic complexity required for effective actuator is to utilize the formulation for purposes of model-based nonlinear control. Nonlinear control charge control. One of the primary reasons for deriving a
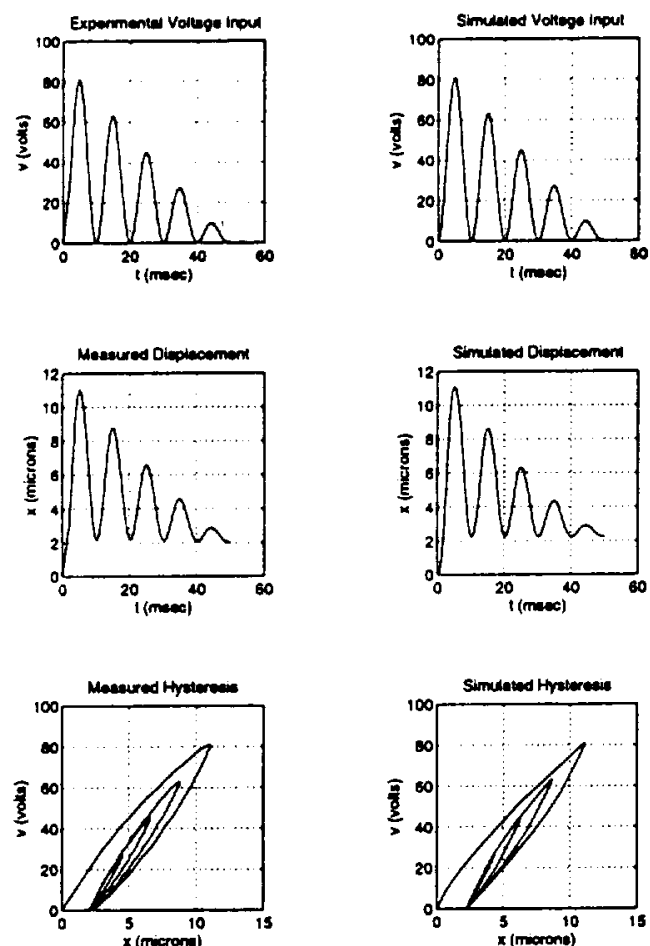

Figure 10. Measured and simulated PZT behavior showing voltage and endpoint displacement for a $100 \mathrm{~Hz}$ linearly decaying sinusoidal voltage input.

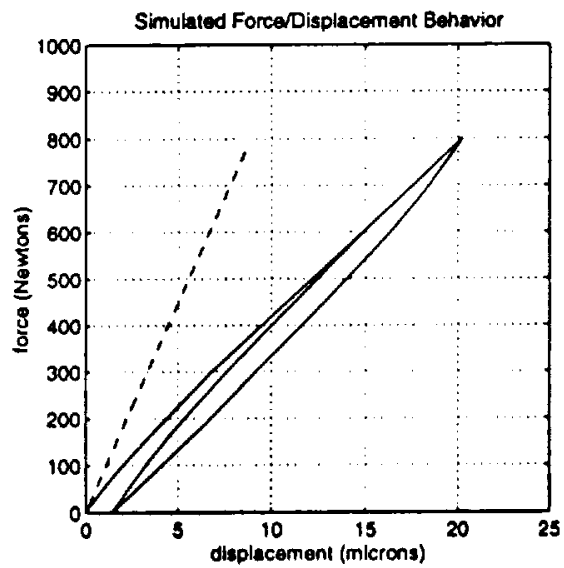

Figure 11. Simulated PZT behavior showing force versus endpoint displacement for a $10 \mathrm{~Hz}$ sinusoidal extcrnal force input for cases with the electrical leads open (dashed line) and shorted (solid line). lumped-parameter real-time description of the PZT techniques provide specific methods of addressing nonlinear hehavior, and can therefore offer a means of achicving stable, high performance voltage control. Such an approach would obviate the need for complex electronic hardware, but would require a more sophisticated controller than a charge control approach and therefore would likely entail a greater computational cost. Ascertaining the specific cost versus benefit of the two approaches will be a topic of future investigations.

\section{Summary and Conclusions}

The model presented accurately represents the behavior of a piezoelectric stack actuator in a lumpedparameter real-time representation, and can therefore be utilized for purposes of model-based control analysis and design. The static hysteresis evinced by the PZT actuator was identified as a rate-independent dissipation and was faithfully represented by a generalized elasto-slip model which was originally formulated by J.C. Maxwell in the nineteenth century. Despite the presence of this nonlinearity, the relationship between charge delivered to the PZT and endpoint displacement of the stack was observed to have simple second-order linear characteristics. Effective closed-loop control of actuator displacement can therefore be achieved either by incorporating a nonlinear controller that commands actuator voltage or by utilizing a linear controller that commands actuator charge.

\section{Acknowledgments}

Support for this work was provided by NASA Grant No. NAGW-4723. The authors gratefully acknowledge this support.

\section{References}

[1] Comstock, R., "Charge Control of Piezoelectric Actuators to Reduce Hysteresis Effects," United States Patent \#4.263,527. Assignee: The Charles Stark Draper Laboratory. Cambridge, MA. 1981.

[2] Lazan, Benjamin J., Damping of Materials and Members in Siructural Mechanics. Pergamon Press, London, 1968.

[3] Newcomb, C. and Flinn, I., "Improving the Linearity of Piezoelectric Ceramic Actuators," Electronics Letters, 1982, Vol. 18, No. 11, May, pp. 442-444.

[4] Standards Committee of the IEEE Ultrasonics, Ferroclectrics, and Frequency Control Socicty, An American Nutional Standard: IEEE Stundard on Piezoelectricity. The Institute of Electrical and Electronics Engineers, 1987. ANSI//EEE Std 176-1987, Now York. 